\title{
Clinical Study \\ Prevalence, Comorbid Associations and Prognostic Value of the Hyperdense Middle Cerebral Artery Sign
}

\author{
Patrick Aouad, ${ }^{1,2}$ Andrew Hughes, ${ }^{1,2}$ Nishant Valecha, ${ }^{2}$ Yash Gawarikar, ${ }^{1,2}$ Kate Ahmad, \\ Ross O'Neil, ${ }^{2,3}$ Murali Guduguntla, ${ }^{3}$ and Christian Lueck ${ }^{1,2}$ \\ ${ }^{1}$ Department of Neurology, The Canberra Hospital, Canberra, ACT 2605, Australia \\ ${ }^{2}$ Australian National University, Canberra, ACT 0200, Australia \\ ${ }^{3}$ Department of Radiology, The Canberra Hospital, Canberra, ACT 2605, Australia
}

Correspondence should be addressed to Patrick Aouad; patrick_aouad@msn.com

Received 6 March 2013; Accepted 4 April 2013

Academic Editors: H. C. Emsley, H. McNaughton, and J. Wambaugh

Copyright (C) 2013 Patrick Aouad et al. This is an open access article distributed under the Creative Commons Attribution License, which permits unrestricted use, distribution, and reproduction in any medium, provided the original work is properly cited.

\begin{abstract}
Background. The significance of the Hyperdense Middle Cerebral Artery Sign (HMCAS) is uncertain. Aims. This prospective study investigated the sensitivity, specificity, prevalence, prognosis, interobserver variability, and associated clinical features of HMCAS in acute ischemic stroke. Methods. Initial CT scans of 117 patients with acute ischemic stroke or transient ischemic attack (TIA) and 65 age-matched controls were reported independently by two neuroradiologists blinded to diagnosis. Details of initial stroke severity and comorbidities were collected, and outcome on the modified Rankin scale (mRS) was assessed at 3-6 months. Results. HMCAS was seen in $15 \%$ of all ischemic strokes and $25 \%$ of all middle cerebral artery (MCA) strokes; specificity was $100 \%$. HMCAS was associated with more severe initial deficit and atrial fibrillation. Only $21 \%$ of patients with a first-ever MCA stroke and HMCAS had a good outcome $(\mathrm{mRS} \leq 2)$ compared to $55 \%$ of those without the sign $(P<0.001)$. Interobserver agreement was only 0.747 (Kappa statistic). Conclusion. The prevalence, specificity, sensitivity, and clinical associations of HMCAS were similar to previous reports. This study confirmed prospectively that HMCAS was associated with a poorer outcome at 3 to 6 months and demonstrated interobserver variability in detection of the sign.
\end{abstract}

\section{Introduction}

The Hyperdense Middle Cerebral Artery Sign (HMCAS) was first described about thirty years ago as a high-density structure within the territory of the middle cerebral artery (MCA) on nonenhanced Computed Tomography (NECT) scans of patients with acute ischemic stroke [1].

A number of investigators have calculated the sensitivity and specificity of the HMCAS, as well as the relationship between the presence of the sign and initial stroke severity [2-6]. Collectively, the published data suggest the sign is highly specific and associated with a more severe initial deficit [7-9]. Associations between the HMCAS and risk factors for ischemic heart disease, hypercoagulopathy, and atrial fibrillation (AF) have also been described [10, 11]. Research has focused predominantly on the prognostic implications in patients who demonstrate an HMCAS on early NECT scans [12-16]. The consensus is that the presence of an HMCAS is associated with a poorer outcome. However, the majority of studies looking at this have been retrospective. There are no published studies that have looked prospectively at the natural history of patients with an HMCAS, that is, without thrombolysis, beyond twenty-one days.

Recent studies have evaluated the outcome of thrombolytic therapy in the subgroup of patients with the HMCAS, and the sign is becoming increasingly important as a clinical marker in the initial management of acute ischemic stroke [17-23]. This initial management is time-critical, but it is entirely dependent on accurate detection of the sign. It is therefore important to determine the accuracy (i.e., interobserver variability) with which the sign can be detected in the setting of standard clinical practice.

This study was set up with three aims. First, we aimed to evaluate the prognostic significance of the HMCAS prospectively at 3 to 6 months following stroke onset. Second, we aimed to assess inter-observer variability in the detection of 
the sign, and, third, we aimed to reassess any associations between the presence of the sign and other clinical features in patients presenting with acute ischemic stroke.

\section{Materials and Methods}

2.1. Study Group. Patients presenting to the Canberra Hospital with acute ischemic stroke or transient ischemic attack (TIA) were recruited prospectively between August 2009, and December 2010. Patients all underwent NECT scanning within 12 hours of arrival. A control group was recruited within the same time frame as the active group and comprised patients admitted to Canberra Hospital for reasons other than stroke or TIA but still requiring NECT scans of the brain.

Patients were excluded from the study if (1) the NECT scan was performed more than 12 hours after the onset of symptoms, (2) the NECT scan was of poor quality due to radiographic artifact (as determined by the reporting neuroradiologists), (3) the patient had significant preexisting intracranial pathology (e.g., tumour or AV malformation), (4) the patient had undergone previous neurosurgery, or (5) the patient was less than 50 years old.

All subjects gave informed consent. The ACT Health Human Research Ethics committee approved this study in May 2009 (ETH. 11.08.1021).

2.2. Clinical Data. Demographic, comorbid, and laboratory data were collected for all stroke and TIA patients from the Canberra Hospital's computerised database. Information collected included a prior history of stroke, transient ischaemic attack (TIA), atrial fibrillation (AF), congestive cardiac failure (CCF), ischemic heart disease (IHD), hypertension, hypercholesterolemia, diabetes, and/or smoking. Laboratory parameters recorded included haematocrit, haemoglobin, the international normalized ratio (INR), and the results of carotid Doppler.

The Scandinavian Stroke Scale (SSS) was used to evaluate initial stroke severity within the emergency department as this was the standard clinical scale used at the Canberra Hospital at the time [24]. The type of stroke was assessed and recorded according to the Oxfordshire Community Stroke Project (OCSP) classification system [25].

A structured telephone interview was used to evaluate patient disability between 3 and 6 months after symptom onset using the modified Rankin scale (mRS) [26].

2.3. CT Data. All NECT scans were performed with multislice spiral CT (Toshiba Aquilion 16 slice) with $1 \mathrm{~mm}$ contiguous axial slices from the occipital foramen to the skull vault with $1 \mathrm{~mm}$ reconstructions.

NECT scans of patients were deidentified and presented independently to two neuroradiologists who were blinded to the diagnosis. The neuroradiologists were asked to report on the presence or absence of ischemic stroke and to indicate whether or not there was an HMCAS using a standardised template designed specifically for this study. An HMCAS was reported as present if the MCA on that side was deemed to be of sufficiently high attenuation relative to (a) the contralateral side, (b) the surrounding parenchyma and (c) the basilar artery. If present, early parenchymal changes associated with cerebral infarction provided supportive information. The neuroradiologists were also asked to comment on whether the scan was inadequate for technical reasons.

2.4. Data Analysis. Inter-observer agreement between the two neuroradiologists was assessed using the Kappa statistic. For the purposes of further analysis an HMCAS was only considered to be present if both radiologists agreed.

Three analyses were performed in order to address the various aims of this study.

Analysis 1. This examined all strokes and TIA patients to determine the prevalence, sensitivity, and specificity of the HMCAS as well as to investigate the presence of any comorbid associations with the sign. For this analysis, sensitivity was defined as the percentage of all strokes with an HMCAS (and, in this case, was therefore the same as prevalence), and specificity reflected the detection of an HMCAS in the control group.

Analysis 2. This analysis was restricted to those patients who were experiencing their first-ever stroke in order to evaluate the relationships between the HMCAS and initial severity and outcome at 3 to 6 months.

Analysis 3. This looked only at patients who had suffered a first-ever ischemic stroke in the MCA territory to allow comparison between patients with and without the HMCAS.

$t$-tests, cross-tabulation, linear regression analysis, and Fisher's exact test were used to evaluate associations between comorbid clinical features, SSS, and mRS and the HMCAS. Associations were considered to be significant if $P<0.05$.

\section{Results}

3.1. Baseline Data. In total, 117 patients with stroke or TIA ( 60 male, 57 female) and 65 control patients ( 28 male, 37 female) were recruited. The mean age of all stroke and TIA patients was $72 \pm 2.6$ years compared to $69 \pm 3.1$ years in the control group (nonsignificant) (Table 1 ).

Of the 117 patients, 17 had TIAs, 61 had MCA territory infarcts, 20 had posterior (i.e., vertebrobasilar territory) circulation infarcts, 6 had infarcts in the anterior cerebral artery territory, 7 had posterior cerebral artery territory events, and 6 had lacunar strokes.

The mean time between stroke or TIA onset and imaging was $4.6 \pm 0.7$ hours. There was no significant difference in mean time from symptom onset to imaging across all groups of patients (Table 1).

Comparison between the two neuroradiologists yielded a Kappa statistic of $0.747 \pm 0.198$.

Three patients within the stroke group were thrombolysed; all three had MCA strokes, one with an HMCAS and the other two without. Inclusion or exclusion of these patients did not alter the results significantly, so thrombolysed patients were included in subsequent analyses. 
TABLE 1: Mean age of patients and controls and time to CT scan.

\begin{tabular}{lccc}
\hline & $N$ & Mean age (years $\left.^{*}\right)$ & Time to scan $\left(\right.$ hours $\left.^{*}\right)$ \\
\hline All patients (strokes and TIAs) & 117 & $72 \pm 2.6$ & $4.6 \pm 0.7$ \\
Strokes & 100 & $72 \pm 2.6$ & $4.6 \pm 0.8$ \\
$\quad$ Middle cerebral artery infarcts & 61 & $72 \pm 0.9$ & $4.7 \pm 1.0$ \\
$\quad$ HMCAS +ve & 15 & $77 \pm 6.3$ & $4.7 \pm 1.1$ \\
$\quad$ HMCAS -ve & 46 & $71 \pm 4.1$ & $4.7 \pm 1.1$ \\
Other strokes & 39 & $72 \pm 4.2$ & $4.9 \pm 1.7$ \\
$\quad$ HMCAS +ve & 0 & - & - \\
$\quad$ HMCAS -ve & 39 & $72 \pm 4.2$ & $4.9 \pm 1.7$ \\
TIAs & 17 & $69 \pm 3.1$ & $4.3 \pm 1.8$ \\
Controls & 65 & & - \\
\hline
\end{tabular}

${ }^{*}$ No statistically significant difference in means between all groups.

Analysis 1. In total, 15 patients were found to have an HMCAS on their emergency NECT. All of them suffered a stroke affecting the middle cerebral artery territory. The prevalence of the HMCAS within the ischemic stroke population $(N=$ 100 , see Table 1) was therefore $15 \%$. No controls were reported to have an HMCAS; that is, the sign had a sensitivity of $15 \%$ and a specificity of $100 \%$.

Comorbid and laboratory data were then analysed as a function of HMCAS. In all, 15 patients were HMCAS +ve, as above, and the remaining 102 patients (comprising 46 HMCAS -ve MCA strokes, 39 other strokes, and 17 TIAs) were HMCAS -ve. The only significant association that correlated positively with the presence of an HMCAS after performing linear regression analysis and applying Fisher's exact test was atrial fibrillation (Table 2).

Analysis 2. After excluding 24 patients with previous stroke from the total of 100 patients with stroke (Table 1 ), a total of 14 patients were HMCAS +ve, and the remainder $(N=62)$ were HMCAS -ve. The initial stroke severity as determined by the SSS was significantly greater in patients with the HMCAS than in those without it $(P=0.002)$ (Table 3$)$.

Similarly, the level of disability as assessed by mRS at 3 to 6 months in patients suffering their first-ever stroke was significantly greater in patients with the HMCAS than in those without it $(P<0.001)$ (Table 3$)$.

Analysis 3. The prevalence of the HMCAS within patients suffering from an infarct in the MCA territory $(N=61)$ was 25\%. 53\% of patients with an HMCAS and a first-ever MCA territory infarct had a diagnosis of AF, compared to only $19 \%$ in those without an HMCAS $(P=0.042)$. It is worth noting that over $70 \%$ of patients who had a history of AF had a subtherapeutic INR (less than 2) (Table 2).

Patients with an HMCAS had significantly more severe initial strokes and poorer outcomes at 3 to 6 months (Table 3 ). Specifically, at 3-6 months only $3(21 \%)$ of all patients presenting with an HMCAS on NECT had an $\mathrm{mRS} \leq 2$ compared to 17 (55\%) in all first-ever MCA territory strokes $(P=0.05)$ (Figure 1).

\section{Discussion}

The results of this prospective observational study have shown that patients presenting with an HMCAS on their
TABLE 2: Clinical features recorded and analysed for all ischemic stroke and TIA patients.

\begin{tabular}{lccc}
\hline $\begin{array}{l}\text { Demographic/ } \\
\text { clinical factor }\end{array}$ & $\begin{array}{c}\text { HMCAS +ve } \\
n=15\end{array}$ & $\begin{array}{c}\text { HMCAS -ve } \\
N=102 \\
n(\%)\end{array}$ & $P^{\dagger}$ \\
\hline Sex (male) & $11(73)$ & $53(52)$ & 0.507 \\
History of TIA & $1(7)$ & $26(25)$ & 0.307 \\
History of stroke & $1(7)$ & $25(25)$ & 0.305 \\
History of AF & $8(53)$ & $19(19)$ & 0.042 \\
History of CCF & 0 & $10(10)$ & 0.606 \\
History of IHD & $2(7)$ & $19(19)$ & 1.000 \\
Hypercholesterolemia & $11(73)$ & $80(78)$ & 1.000 \\
Hypertension & $9(60)$ & $76(75)$ & 0.667 \\
Smoking & $3(20)$ & $24(24)$ & 1.000 \\
Diabetes & $2(20)$ & $16(16)$ & 1.000 \\
High hematocrit & 0 & $4(4)$ & 1.000 \\
High hemoglobin & 0 & $5(5)$ & 1.000 \\
Subtherapeutic INR & $6(75)$ & $14(73)$ & 1.000 \\
(patients with AF) & & $9(9)$ & 0.099 \\
Significant carotid & $4(27)$ & & \\
stenosis & & &
\end{tabular}

${ }^{\dagger}$ Fisher's exact test (two-tailed) $P$ value compares the proportion with risk factor to the presence of an HMCAS.

emergency NECT brain scan are highly likely to be suffering from ischemic stroke affecting the middle cerebral artery territory. These patients are more likely to exhibit more severe initial clinical deficit, to have atrial fibrillation, and to be severely disabled or dead within 3 to 6 months following their stroke than stroke patients without the sign.

There were no significant differences between groups in terms of baseline demographic data or the time between symptom onset and NECT scan. Agreement between radiologists on the presence of an HMCAS was good but not excellent, according to the Kappa statistic [27]. This level of discrepancy between specialist radiologists has been reported previously and is not that surprising given the subjective nature of reporting [28-30]. The possibility of disagreement raises concern in relation to the possible role of the sign in making time-critical clinical judgments such as whether or not to thrombolyse a patient, particularly because the scans 
TABLE 3: Initial deficit and disability associated with the presence of an HMCAS on NECT.

\begin{tabular}{|c|c|c|c|}
\hline & $\begin{array}{c}\text { First-ever } \\
\text { strokes } \\
\text { HMCAS -ve } \\
N=62\end{array}$ & $\begin{array}{c}\text { First-ever } \\
\text { strokes } \\
\text { HMCAS +ve } \\
N=14\end{array}$ & $\begin{array}{c}\text { Significance }^{\ddagger} \\
P\end{array}$ \\
\hline \multicolumn{4}{|l|}{ Initial severity } \\
\hline$\left(\right.$ mean $\left.\mathrm{SSS}^{\S}\right)$ & $46.2 \pm 3.15$ & $27.9 \pm 7.64$ & 0.002 \\
\hline \multicolumn{4}{|c|}{ Disability at 3-6 months } \\
\hline (mean mRS) & $2.0 \pm 0.45$ & $4.4 \pm 1.1$ & $<0.001$ \\
\hline \multirow[t]{4}{*}{$\mathrm{mRS}^{\|} \leq 2: n(\%)$} & $40(65)$ & $3(21)$ & $<0.001$ \\
\hline & $\begin{array}{c}\text { First-ever } \\
\text { MCA } \\
\text { strokes }\end{array}$ & $\begin{array}{c}\text { First-ever } \\
\text { MCA } \\
\text { strokes }\end{array}$ & \\
\hline & HMCAS -ve & HMCAS +ve & \\
\hline & $N=31$ & $N=14$ & \\
\hline \multicolumn{4}{|l|}{ Initial severity } \\
\hline$\left(\right.$ mean SSS $\left.{ }^{\S}\right)$ & $43.6 \pm 3.47$ & $27.9 \pm 7.64$ & 0.006 \\
\hline \multicolumn{4}{|c|}{ Disability at $3-6$ months } \\
\hline (mean mRS) & $2.6 \pm 0.67$ & $4.4 \pm 1.1$ & 0.008 \\
\hline $\mathrm{mRS}^{\|} \leq 2: n(\%)$ & $17(55)$ & $3(21)$ & 0.05 \\
\hline
\end{tabular}

${ }^{\ddagger}$ Two-tailed $t$-test (SSS) and Fisher's exact test after linear regression analysis.

${ }^{\S}$ SSS: Scandinavian Stroke Score [24]. Range from 2 in a maximally-impaired patient to 58 in a normal person.

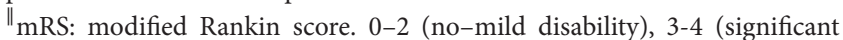
disability), and 5-6 (high level disability or death). Dichotomized $\mathrm{mRS} \leq 2$ as favourable and $m R S \geq 2$ as poor outcome.

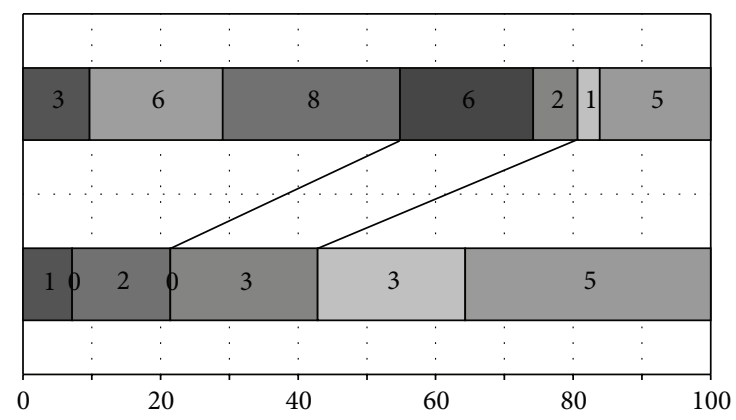

(\%)

$\begin{array}{ll}\square \text { mRS } 0 & \square \text { mRS 4 } \\ \square \text { mRS 1 } & \square \text { mRS 5 } \\ \square \text { mRS 2 } & \square \text { mRS 6 } \\ \square \text { mRS 3 } & \end{array}$

FIGURE 1: Modified Rankin scale outcomes of 31 first-ever MCA territory strokes without an HMCAS (upper bar) and 14 first-ever MCA territory strokes with an HMCAS (lower bar).

will be read by doctors with considerably less experience than the neuroradiologists who reported the scans in the present study.

We found no false positives in this study. A small number have been described in the literature and it has been suggested that these may relate to inter-observer variability or, alternatively, to an elevated hematocrit and/or conditions that promote vascular calcification [31].

After excluding all the patients who had previously suffered a stroke, those with an HMCAS had a more severe initial deficit. Restricting the analysis to new strokes within the MCA territory alone did not alter this trend. Our results also clearly demonstrate that patients with an HMCAS have a poorer outcome between 3 and 6 months after stroke as assessed by the mRS.

Atrial fibrillation is known to be associated with more severe ischemic stroke $[32,33]$. In this study, AF was significantly more common in patients with an HMCAS on NECT. This has been reported previously and is consistent with the fact that the red blood cell content of the thrombus is likely to be higher in clot associated with atrial fibrillation [34].

The role of the HMCAS in contributing to the decision of whether or not to thrombolyse a patient with an acute ischemic stroke has been the subject of a number of studies, but the results remain inconclusive [35-37]. Some authors have suggested a need for more effective, novel, and potentially aggressive revascularization techniques in these patients $[38,39]$. Either way, it is important that the sign should be detected accurately at the time of diagnosis, and our finding of less than $100 \%$ agreement between experts casts doubt on the ability of nonexperts to detect the presence or absence of the sign accurately. Some work has been done on this previously, suggesting that HMCAS sensitivity could be enhanced by changing the parameters of the CT scanning [40-42]. However, this is unlikely to become standard practice when scanning patients in emergency departments, and further work in this area is clearly necessary.

This study has a number of limitations. First, the numbers are small and, while the subjects were recruited prospectively, much of the data relating to clinical associations was assessed retrospectively from patient charts. It is also worth noting that lacunar strokes are difficult to diagnose clinically, but were included as per the OCSP classification system definition. Second, stroke severity was assessed using the Scandinavian Stroke Scale rather than the more commonly-used $\mathrm{NIH}$ stroke scale. This was due to the fact that the SSS was the standard clinical tool used in the department at the time of the study. While acknowledging all these possible limitations, we do not believe they would have a significant impact on the conclusions.

In conclusion, this study had three aims. First, we have demonstrated prospectively that the untreated outcome at 3 to 6 months is significantly worse in patients with the HMCAS than those without it. Second, we have demonstrated that the level of agreement between expert neuroradiologists when looking at standard clinical scans is only 0.747. Third, we have confirmed an association between the presence of the sign with both atrial fibrillation and a more severe stroke. Because it has been suggested that the sign may be useful in determining the role of thrombolysis in the acute setting, more work is needed, particularly with a view to investigating the reliability of detection of the sign and so reducing the possibility of inter-observer variability.

\section{Conflict of Interests}

There is no conflict of interests. 


\section{References}

[1] G. Gacs, A. J. Fox, H. J. M. Barnett, and F. Vinuela, "CT visualization of intracranial arterial thromboembolism," Stroke, vol. 14, no. 5, pp. 756-762, 1983.

[2] T. A. Tomsick, T. G. Brott, C. P. Olinger et al., "Hyperdense middle cerebral artery: incidence and quantitative significance," Neuroradiology, vol. 31, no. 4, pp. 312-315, 1989.

[3] T. A. Tomsick, T. G. Brott, A. A. Chambers et al., "Hyperdense middle cerebral artery sign on CT: efficacy in detecting middle cerebral artery thrombosis," American Journal of Neuroradiology, vol. 11, no. 3, pp. 473-477, 1990.

[4] T. Moulin, F. Cattin, T. Crépin-Leblond et al., "Early CT signs in acute middle cerebral artery infarction: predictive value for subsequent infarct locations and outcome," Neurology, vol. 47, no. 2, pp. 366-375, 1996.

[5] T. Tomsick, T. Brott, W. Barsan, J. Broderick, E. C. Haley, and J. Spilker, "Thrombus localization with emergency cerebral CT," American Journal of Neuroradiology, vol. 13, no. 1, pp. 257-263, 1992.

[6] T. Goda, "Computed tomographic parameters predicting neurological deterioration in patients with middle cerebral artery territory infarction," Egyptian Journal of Neurology, Psychiatry and Neurosurgery, vol. 47, no. 4, pp. 563-568, 2010.

[7] S. Bastianello, A. Pierallini, C. Colonnese et al., "Hyperdense middle cerebral artery CT sign. Comparison with angiography in the acute phase of ischemic supratentorial infarction," Neuroradiology, vol. 33, no. 3, pp. 207-211, 1991.

[8] D. Leys, J. P. Pruvo, O. Godefroy, and X. Leclerc, "Prevalence and significance of hyperdense middle cerebral artery in acute stroke," Stroke, vol. 23, no. 3, pp. 317-324, 1992.

[9] T. Moulin, F. Cattin, T. Crépin-Leblond et al., "Early CT signs in acute middle cerebral artery infarction: predictive value for subsequent infarct locations and outcome," Neurology, vol. 47, no. 2, pp. 366-375, 1996.

[10] E. Berge, P. H. Nakstad, and P. M. Sandset, "Large middle cerebral artery infarctions and the hyperdense middle cerebral artery sign in patients with atrial fibrillation," Acta Radiologica, vol. 42, no. 3, pp. 261-268, 2001.

[11] M. Zorzon, G. Mase, F. Pozzi-Mucelli et al., "Increased density in the middle cerebral artery by nonenhanced computed tomography: prognostic value in acute cerebral infarction," European Neurology, vol. 33, no. 3, pp. 256-259, 1993.

[12] Y. Launes and L. Ketonen, "Dense middle cerebral artery sign: an indicator of poor outcome in middle cerebral artery area infarction," Journal of Neurology Neurosurgery and Psychiatry, vol. 50, no. 11, pp. 1550-1552, 1987.

[13] S. Ricci, N. Caputo, G. Aisa et al., "Prognostic value of the dense middle cerebral artery sign in patients with acute ischemic stroke," The Italian Journal of Neurological Sciences, vol. 12, no. 1, pp. 45-47, 1991.

[14] R. Von Kummer, U. Meyding-Lamade, M. Forsting et al., "Sensitivity and prognostic value of early CT in occlusion of the middle cerebral artery trunk," American Journal of Neuroradiology, vol. 15, no. 1, pp. 9-15, 1994.

[15] D. M. Somford, P. J. Nederkoorn, D. R. Rutgers, L. J. Kappelle, W. P. T. M. Mali, and J. Van der Grond, "Proximal and distal hyperattenuating middle cerebral artery signs at CT: different prognostic implications," Radiology, vol. 223, no. 3, pp. 667-671, 2002.

[16] A. Hengirmen, M. Neyal, A. Srikci, A. Neyal, M. Gur, and N. Tahtaci, "Hyperdense middle cerebral artery sign does not always predict a poor prognosis," International Medical Journal, vol. 9, no. 3, pp. 205-208, 2002.

[17] T. Tomsick, T. Brott, W. Barsan et al., "Prognostic value of the hyperdense middle cerebral artery sign and stroke scale score before ultraearly thrombolytic therapy," American Journal of Neuroradiology, vol. 17, no. 1, pp. 79-85, 1996.

[18] C. Manelfe, V. Larrue, R. Von Kummer et al., "Association of hyperdense middle cerebral artery sign with clinical outcome in patients treated with tissue plasminogen activator," Stroke, vol. 30, no. 4, pp. 769-772, 1999.

[19] P. Agarwal, S. Kumar, S. Hariharan et al., "Hyperdense middle cerebral artery sign: can it be used to select intra-arterial versus intravenous thrombolysis in acute ischemic stroke?" Cerebrovascular Diseases, vol. 17, no. 2-3, pp. 182-190, 2004.

[20] M. J. H. Aries, M. Uyttenboogaart, K. Koopman et al., "Hyperdense middle cerebral artery sign and outcome after intravenous thrombolysis for acute ischemic stroke," Journal of the Neurological Sciences, vol. 285, no. 1-2, pp. 114-117, 2009.

[21] T. Kharitonova, M. Thorén, N. Ahmed et al., "Disappearing hyperdense middle cerebral artery sign in ischaemic stroke patients treated with intravenous thrombolysis: clinical course and prognostic significance," Journal of Neurology, Neurosurgery and Psychiatry, vol. 80, no. 3, pp. 273-278, 2009.

[22] A. I. Qureshi, M. A. Ezzeddine, A. Nasar et al., "Is IV tissue plasminogen activator beneficial in patients with hyperdense artery sign?” Neurology, vol. 66, no. 8, pp. 1171-1174, 2006.

[23] C. Nichols, J. Khoury, T. Brott, and J. Broderick, "Intravenous recombinant tissue plasminogen activator improves arterial recanalization rates and reduces infarct volumes in patients with hyperdense artery sign on baseline computed tomography," Journal of Stroke and Cerebrovascular Diseases, vol. 17, no. 2, pp. 64-68, 2008.

[24] E. Aberg, G. Adielsson, and A. Almqvist, "Multicenter trial of hemodilution in ischemic stroke-background and study protocol," Stroke, vol. 16, no. 5, pp. 885-890, 1985.

[25] J. Bamford, P. Sandercock, M. Dennis, C. Warlow, and J. Burn, "Classification and natural history of clinical identifiable subtypes of cerebral infarction," The Lancet, vol. 337, no. 8756, pp. 1521-1526, 1991.

[26] R. Bonita and R. Beaglehole, "Recovery of motor function after stroke," Stroke, vol. 19, no. 12, pp. 1497-1500, 1988.

[27] J. R. Landis and G. G. Koch, "The measurement of observer agreement for categorical data," Biometrics, vol. 33, no. 1, pp. 159-174, 1977.

[28] T. A. Tomsick, T. G. Brott, A. A. Chambers et al., "Hyperdense middle cerebral artery sign on CT: efficacy in detecting middle cerebral artery thrombosis," American Journal of Neuroradiology, vol. 11, no. 3, pp. 473-477, 1990.

[29] R. Von Kummer, R. Holle, U. Grzyska et al., "Interobserver agreement in assessing early CT signs of middle cerebral artery infarction," American Journal of Neuroradiology, vol. 17, no. 9, pp. 1743-1748, 1996.

[30] K. Abul, E. Selariu, M. Brizzi, and J. Petersson, "Hyperdense middle cerebral artery sign in multidetector computed tomography: definition, occurrence, and reliability analysis," Neurology India, vol. 57, no. 2, pp. 143-150, 2009.

[31] R. A. Rauch, C. Bazan, E. M. Larsson, and J. R. Jinkins, "Hyperdense middle cerebral arteries identified on CT as a false sign of vascular occlusion," American Journal of Neuroradiology, vol. 14, no. 3, pp. 669-673, 1993. 
[32] D. A. Dulli, H. Stanko, and R. L. Levine, "Atrial fibrillation is associated with severe acute ischemic stroke," Neuroepidemiology, vol. 22, no. 2, pp. 118-123, 2003.

[33] K. Kimura, K. Minemaisu, and T. Yamaguchi, "Atrial fibrillation as a predictive factor for severe stroke and early death in 15 831 patients with acute ischaemic stroke," Journal of Neurology, Neurosurgery and Psychiatry, vol. 76, no. 5, pp. 679-683, 2005.

[34] D. S. Liebeskind, N. Sanossian, W. H. Yong et al., "CT and MRI early vessel signs reflect clot composition in acute stroke," Stroke, vol. 42, no. 5, pp. 1237-1243, 2011.

[35] D. Georgiadis, F. Wirz, H. C. Von Büdingen et al., "Intravenous thrombolysis in stroke patients with hyperdense middle cerebral artery sign," European Journal of Neurology, vol. 16, no. 2, pp. 162-167, 2009.

[36] J. Rocha, R. Carvalho, F. Sousa et al., "Hyperdensemiddle cerebral artery prognostic value in patients with ischemic stroke submitted to IV trombolysis," Cerebrovascular Diseases, vol. 29, p. 269, 2010.

[37] P. R. Paliwal, A. Ahmad, S. Liang et al., "Persistence of hyperdense middle cerebral artery sign on follow-up ct scan is associated with poor outcome in ischemic stroke patients treated with intravenous thrombolysis," Stroke, vol. 42, no. 3, article e279, 2011.

[38] K. Abul-Kasim, M. Brizzi, and J. Petersson, "Hyperdense middle cerebral artery sign is an ominous prognostic marker despite optimal workflow," Acta Neurologica Scandinavica, vol. 122, no. 2, pp. 132-139, 2010.

[39] J. Eggers and F. Roessler :, "Proximal middle cerebral artery mainstem occlusion: might the combination of intravenous thrombolysis and ultrasound (sonothrombolysis) serve as an alternative to intra-arterial thrombolysis?" Cerebrovascular Diseases, vol. 31, p. 24, 2011.

[40] E. Y. Kim, E. Yoo, H. Y. Choi, J. W. Lee, and J. H. Heo, "Thrombus volume comparison between patients with and without hyperattenuated artery sign on CT," American Journal of Neuroradiology, vol. 29, no. 2, pp. 359-362, 2008.

[41] C. H. Riedel, U. Jensen, A. Rohr et al., "Assessment of thrombus in acute middle cerebral artery occlusion using thin-slice nonenhanced computed tomography reconstructions," Stroke, vol. 41, no. 8, pp. 1659-1664, 2010.

[42] C. H. Riedel, U. Jensen, and O. Jansen :, "Assessing clot burden with thin slice ct to predict failing recanalization in systemic thombolysis in acute stroke," Cerebrovascular Diseases, vol. 29, p. $77,2010$. 


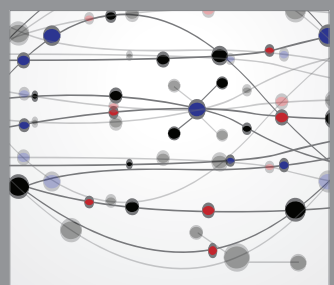

The Scientific World Journal
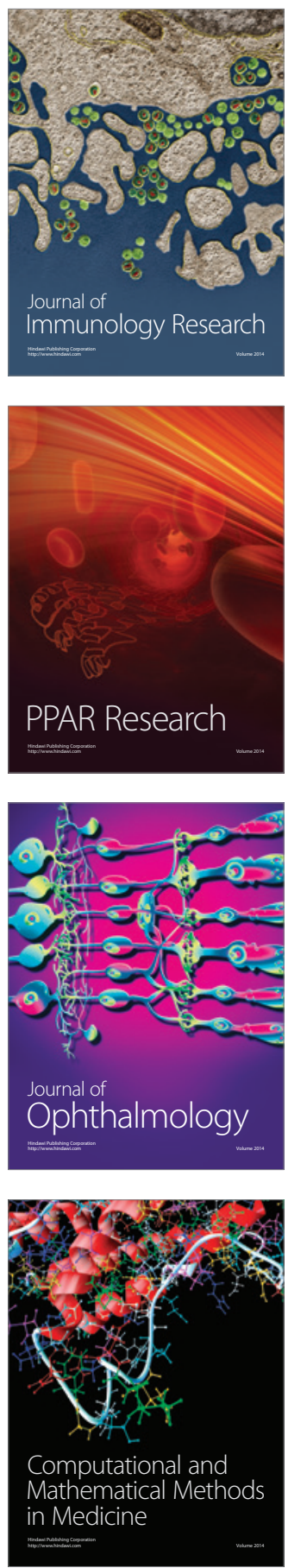

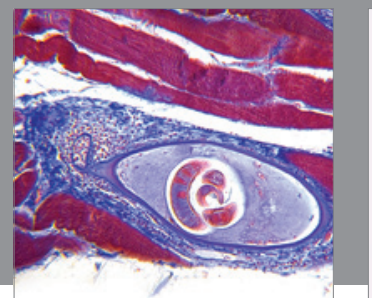

Gastroenterology

Research and Practice
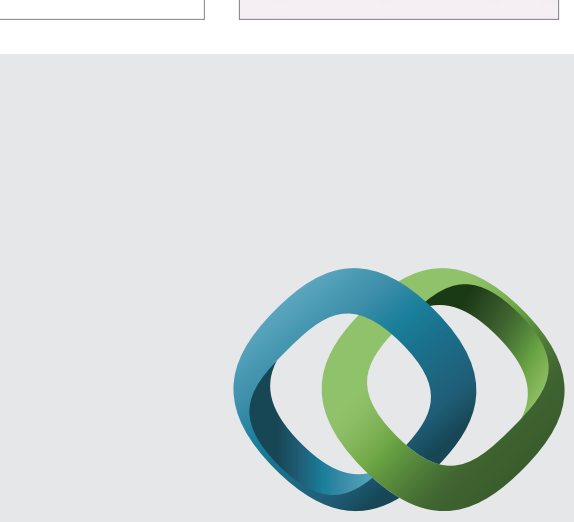

\section{Hindawi}

Submit your manuscripts at

http://www.hindawi.com
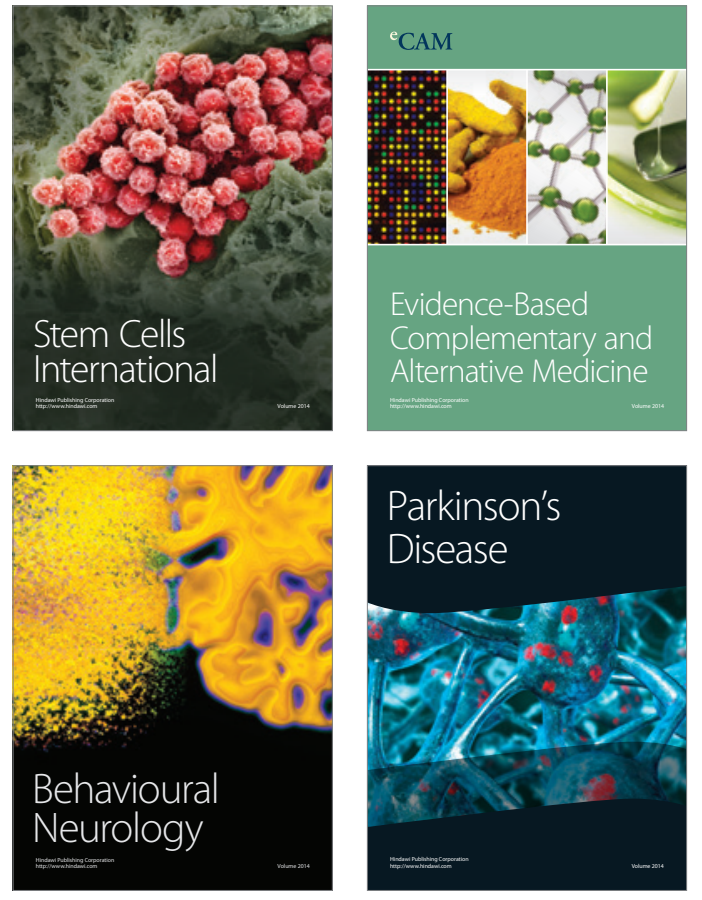
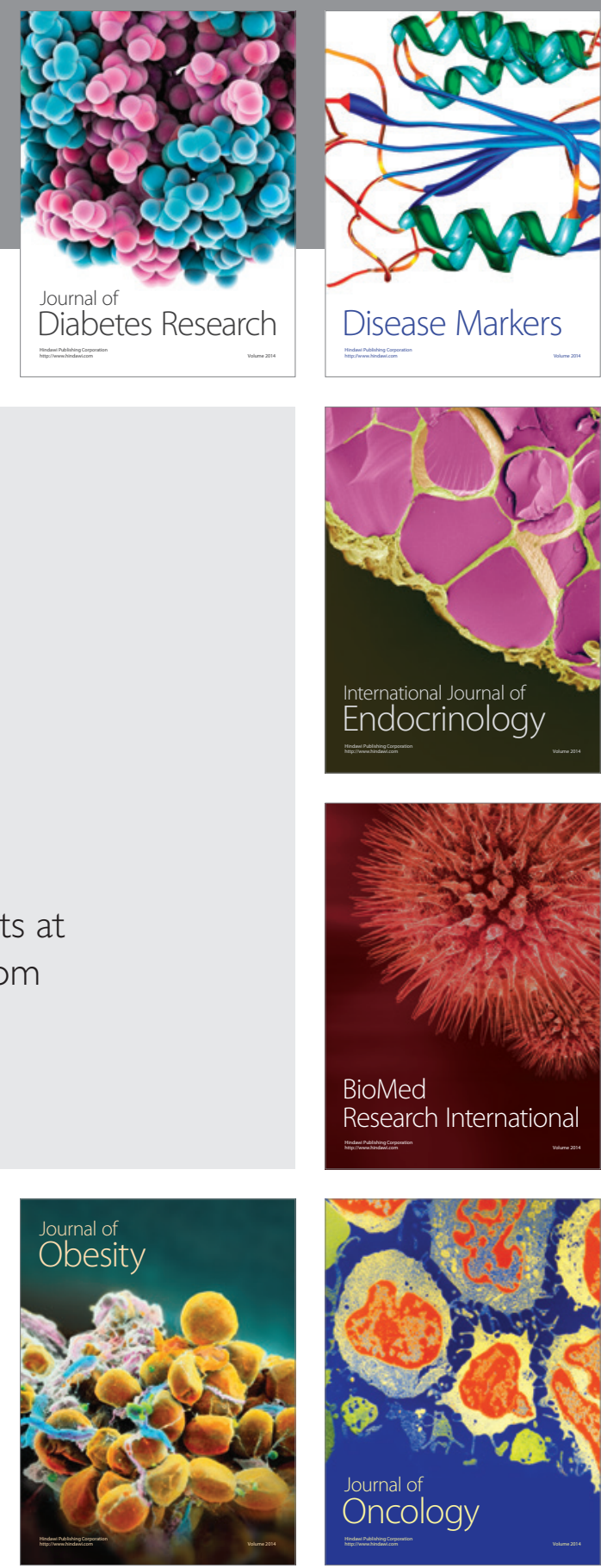

Disease Markers
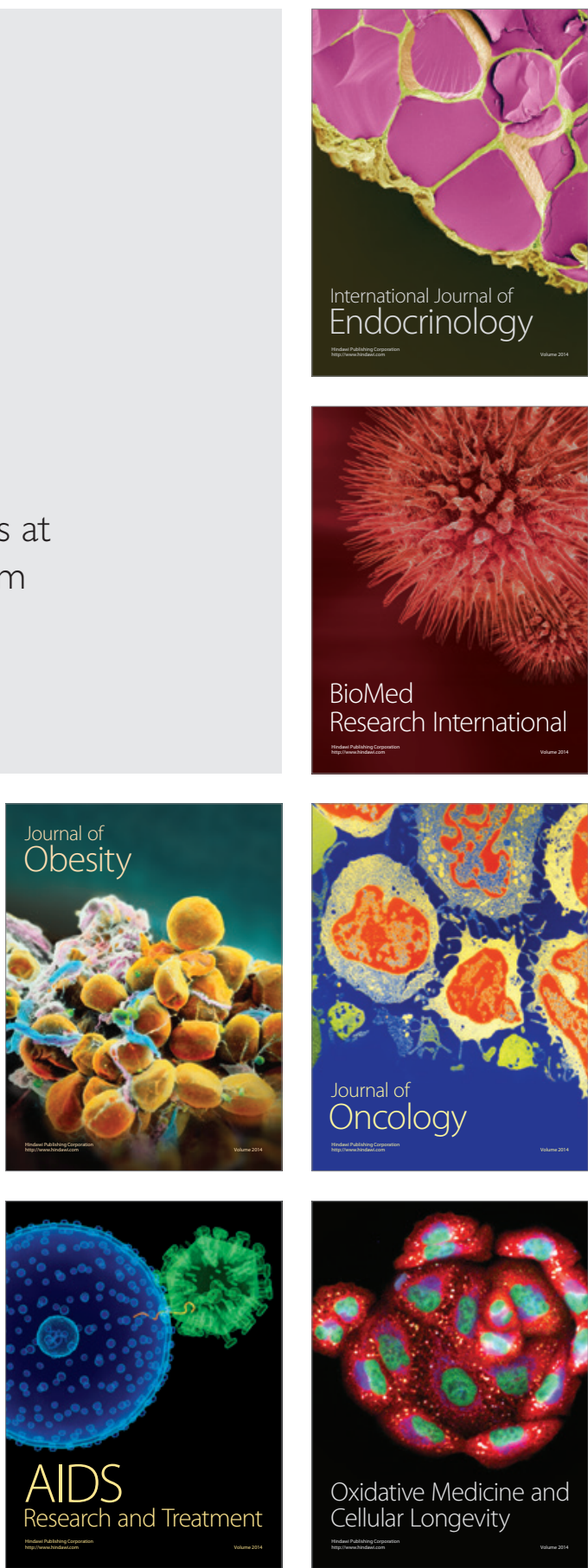